\title{
Relación del soporte familiar y social en el cumplimiento del tratamiento de pacientes con tuberculosis pulmonar en centros de salud
}

\author{
Luz Nancy del Pilar Quevedo Cruz ${ }^{1, a}$, Ruth Liliana Sánchez Requín ${ }^{1, a}$, Fiorella Katherine Villalba Porras \\ 1,a, Doris Velásquez Carranza $2, \mathrm{b,c}$
}

\section{RESUMEN}

Objetivos: Determinar la relación del soporte familiar y social en el cumplimento del tratamiento de pacientes con Tuberculosis Pulmonar. Material y métodos: tipo de estudio descriptivo de corte transversal, correlacional. La población estuvo conformada por 52 pacientes que estuvieron registrados en la Estrategia Sanitaria Nacional de Prevención y Control de la Tuberculosis en los Centros de Salud: Flor de Amancaes y San Juan de Amancaes. Se utilizó como técnica la encuesta, a través de dos cuestionarios validados por juicio de expertos, instrumentos que midieron el soporte familiar y social en el cumplimiento del tratamiento. Resultados: Un $81,8 \%$ de los pacientes que recibieron nivel alto de soporte familiar y social presentaron cumplimiento del tratamiento, mientras que un 36.4\%, que recibieron bajo nivel de soporte familiar y social presentaron ¿cumplimiento? del tratamiento. La diferencia encontrada resultó estadísticamente significativa $(\mathrm{p}=0,034)$. Mientras que un $59.6 \%$ de los pacientes cumplen con el tratamiento, y un 40,4\% no lo cumplen. Conclusiones: el soporte familiar y social es un eje importante en la recuperación del paciente, ya que el paciente se siente más confiado y motivado a culminar con su tratamiento para recuperarse pronto y reinsertarse a su vida cotidiana.

PALABRAS CLAVE: tuberculosis pulmonar, soporte familiar y social, cumplimiento.

\section{Relationship of family and social support in implementing the treatment of patients with pulmonary tuberculosis in health}

\begin{abstract}
SUMMARY
Objectives: To determine the relationship of family and social support in the treatment compliance of patients with pulmonary tuberculosis. Material and methods: The study was a descriptive type of cross-sectional correlation of the 52 patients who were registered in the National Health Strategy for Prevention and Control of Tuberculosis in Health Centers: "Flor de Amancaes" and "San Juan de Amancaes." Data collection: A survey was used as a technique and the survey was conducted through two questionnaires and validated by an expert judgment. The instruments that measure family and social support and adherence to treatment of patients with pulmonary tuberculosis have acceptable level's of reliability. Results: $81.8 \%$ of the patients who received a high level of family and social support showed compliance, while $36.4 \%$ received a low level of family and social support showed compliance. The difference found was statistically significant $(\mathrm{p}=0.034) .59 .6 \%$ of the patients complied with treatment, while 40.4\% did not. Conclusions: family and social support is an important factor in the patient recovery axis, since the patient will feel more confident and motivated to finish the treatment and recover soon and reintegrate into their daily lives.
\end{abstract}

KEYWORDS: Pulmonary tuberculosis, family and social support, compliance.

Facultad de Enfermería, Universidad Peruana Cayetano Heredia. Lima, Perú.

Licenciada en Enfermería ; ${ }^{b}$ Docente Asociada ; ${ }^{\mathrm{c}}$ Magister en Enfermería. 


\section{INTRODUCCIÓN}

La Tuberculosis Pulmonar (TBP) es un problema de salud pública, no solamente en el país sino también a nivel mundial, representa la segunda causa mundial de mortalidad, después del SIDA. Según el último informe de la Organización Mundial de la Salud (OMS) del 2012 existe una disminución continua del número de personas que contraen la enfermedad, registrándose así 8,6 millones para el 2012 (1), más de un tercio de los 77 900 casos nuevos de TB registrados en los países andinos de América del Sur, ocurrieron en Perú (30 000; 39\%), ocupando el segundo lugar en las Américas, seguido por Colombia (16 000; 21\%). La mayor incidencia estimada de TB por 100000 habitantes correspondió en el 2011 a Bolivia (129 casos) y Perú (101 casos), donde había disminuido sustancialmente en los 20 últimos años. La incidencia también descendió en Ecuador y Colombia y fue constante en un nivel inferior en Venezuela (2).

En el año 2009 en Lima, se identificaron 15 distritos con mayor carga de tuberculosis debido a su alta tasa de morbilidad, siendo 8 los principales: El Agustino $(388,4)$, La Victoria (383,1), Santa Anita (282,6), Ate (250,9), San Juan de Lurigancho (212,0), Lima cercado (207,4), Rímac $(203,8)$ y Lince $(203,3)(3)$.

Durante el año 2011, la tasa de morbilidad por TB en Lima Ciudad, Lima Este y Lima Sur fue de 140,6, 196,9 y 142,4 por cada cien mil habitantes respectivamente (4). La tasa de abandonos en el 2010 fue del 5,8\%, el cual ha descendido en comparacióncon el año 2009, 6,3\% (5). Por otro lado cabe mencionar que la familia es el elemento de la estructura de la sociedad responsable de la reproducción biológica y social del ser humano, eje fundamental para motivar al paciente a tomar el tratamiento completo contra la tuberculosis hasta su total restablecimiento, de acuerdo a las indicaciones del personal de salud y realizando los controles médicos respectivos (6).

El apoyo afectivo es esencial ya que permite acompañar al paciente en el curso de la enfermedad, evitando pensamientos de negatividad y baja autoestima. La tuberculosis pulmonar se acompaña de un debilitamiento general, con poca energía y capacidad física para las actividades cotidianas, este debilitamiento se asocia a diferentes niveles de discapacidad por parte de los pacientes,y el estigma social causa un gran impacto en el paciente; es por ello, que se siente rechazado y excluido de la sociedad, deteriorando su autoestima. Esta interpretación representa la "marca o huella" que se impregna en el paciente desde el momento en que se diagnostica la enfermedad (7).

El tratamiento se basa en diversos regímenes de terapia combinada (varios medicamentos) de corta duración formulados en los decenios de 1970 y 1980 y que han ido mejorando en el transcurso de los años (8).

La problemática social de esta enfermedad actualmente se centra en el riesgo de incumplimiento terapéutico y su posible influencia negativa tanto en la salud del paciente como en el control de la enfermedad desde el punto de vista de la salud pública (9).

Así mismo, sabiendo que el entorno afectivo y social (conyugue o compañero (a), hijos, miembros de la familia o parientes, amigos, vecinos), que rodea al paciente influye sobre las decisiones que tomaría con respecto al cumplimiento del tratamiento de la enfermedad (7). El personal de Enfermería se encuentra en mayor contacto con el paciente durante todo el proceso de su tratamiento y; por ello, contará con la mejor oportunidad de conversar con él sobre su enfermedad, reacciones adversas, controles mensuales, y el seguimiento luego del alta (8).

La entrevista es el momento clave para que el personal enfermero se comunique con la persona que presenta la enfermedad de Tuberculosis Pulmonar y esta se realiza con la finalidad de conocer cómo, dónde y cuándo se produjo el contagio (antecedentes epidemiológicos); quienes son sus contactos intra y extrafamiliares, cuáles son las probables causas que facilitaron la adquisición de la enfermedad (condiciones y hábitos de vida, enfermedades paralelas y otros); sobretodo es el momento de escuchar sus necesidades (dudas, temores, angustias); así como de brindarle información sobre la enfermedad y explicarle cómo se va a trabajar en conjunto para llegar al éxito de su tratamiento (8).

El objetivo del presente estudio fue determinar la relación del soporte familiar y social en el cumplimento del tratamiento de pacientes con tuberculosis pulmonar.

\section{MATERIAL Y MÉTODOS}

Estudio descriptivo, correlacional de corte transversal; La población estuvo constituida por 70pacientes de la Estrategia Sanitaria Nacional de Prevención y Control de Tuberculosis de los Centros de Salud Flor de Amancaes y San Juan de Amancaes del distrito del Rímac. El tamaño muestral para el presente estudio fue de 52 pacientes. 


\section{Criterios de selección}

- Pacientes con diagnóstico de Tuberculosis Pulmonar pertenecientes al esquema de tratamiento 1 (1 ra y 2 da fase).

- Pacientes de ambos sexos.

- Pacientes mayores de 18 años.

- Pacientes que acudan a recibir su tratamiento.

- Pacientes que vivan bajo el mismo techo con personas y que compartan el lazo consanguíneo.

Para el estudio se utilizó como instrumento dos cuestionarios

El primer cuestionario consta de 10 preguntas con respuesta dicotómica $\mathrm{Si} / \mathrm{No}$, relacionadas con el soporte familiar y social con el que cuenta el paciente con Tuberculosis Pulmonar.

El segundo cuestionario consta de 10 preguntas con respuesta dicotómica $\mathrm{Si} / \mathrm{No}$ que evalúa el cumplimiento del tratamiento.

Los instrumentos se validaron mediante un juicio de expertos, cuyas opiniones y recomendaciones sirvieron para mejorar los instrumentos planteados. Para el primer instrumento, se obtuvo un $\mathrm{p}=0,0317$ y para el segundo instrumento un $\mathrm{p}=0,0454$.

La prueba piloto se realizó en el Centro de Salud. "Ciudad y Campo" perteneciente a la Red Rímac-SM-LO a 20 pacientes de la Estrategia Sanitaria de Prevención y Control de la Tuberculosis, ya que cuenta con las mismas características sociodemográficas que los centros de salud participantes del estudio.

Para determinar las escalas de clasificación de los instrumentos Soporte familiar y social y Cumplimiento del tratamiento se aplicó la técnica de Estaninos con la muestra piloto en mención, clasificándose los puntajes en los niveles alto, medio y bajo.

Los instrumentos de recolección de datos (cuestionarios), se aplicaron en el mes de noviembre y diciembre del 2013, de lunes a sábado en turnos de mañana y tarde con un promedio de 6 encuestas diarias.

Finalmente, se repartieron las encuestaslos cuestionarios a los pacientes y al mismo tiempo se realizaron visitas domiciliarias para captar a aquellos pacientes que se encontraban en abandono del tratamiento.
Concluida la recolección de información, éstos fueron codificados e ingresados a una base de datos creada en el programa estadístico SPSS versión 19.0.

\section{RESULTADOS}

Los resultados obtenidos se pueden observar en las tablas 1,2 y 3 .

Tabla 1. Soporte familiar y social según el cumplimiento del tratamiento de pacientes con tuberculosis pulmonar en centros de salud del distrito del rimac

Noviembre-diciembre 2013

\begin{tabular}{lrrr}
\hline $\begin{array}{l}\text { SOPORTE } \\
\text { FAMILIAR Y } \\
\text { SOCIAL }\end{array}$ & \multicolumn{2}{c}{ CUMPLIMIENTO } & \multirow{2}{*}{ Total } \\
\cline { 2 - 3 } ALTO & \multicolumn{1}{c}{ SI } & NO & \\
& 18 & 4 & 22 \\
MEDIO & $81,80 \%$ & $18,80 \%$ & $100,00 \%$ \\
& 9 & 10 & 19 \\
BAJO & $47,40 \%$ & $52,60 \%$ & $100,00 \%$ \\
& 4 & 7 & 11 \\
Total & $36,40 \%$ & $63,60 \%$ & $100,00 \%$ \\
& 31 & 21 & 52 \\
& $59,60 \%$ & $25,00 \%$ & $100,00 \%$ \\
\hline
\end{tabular}

Tabla 2. Soporte familiar y social en pacientes con tuberculosis pulmonar en centros de salud del distrito del Rimac. Noviembre-diciembre 2013

\begin{tabular}{lcc}
\hline NIVEL & NUMERO & \% \\
\hline ALTO & 22 & 42,3 \\
MEDIO & 19 & 36,5 \\
BAJO & 11 & 21,2 \\
Total & 52 & 100,0 \\
\hline
\end{tabular}

Tabla 3. Cumplimiento del tratamiento en pacientes con tuberculosis pulmonar en centros de salud del distrito del Rimac. Nov-dic 2013

\begin{tabular}{crr}
\hline NIVEL & NUMERO & \% \\
\hline SI & 31 & 59,6 \\
NO & 21 & 40,4 \\
Total & 52 & 100 \\
\hline
\end{tabular}




\section{DISCUSIÓN}

El estudio se realizó con el objetivo de encontrar la relación entre el soporte familiar y social en el cumplimiento del tratamiento de los pacientes con Tuberculosis Pulmonar. Las características sociodemográficas de la población estuvieron distribuidas de la siguiente manera:

Un $40,4 \%$ de los pacientes encuestados pertenecen al grupo etario de 25 a 44 años de edad y un $71,2 \%$ lo representa el sexo masculino. Con respecto al grado de instrucción se observó que un 53\% contó con estudios secundarios completos y un $1.9 \%$ con estudios universitarios.

En cuanto a la edad, se observóque el grupo etario de 25 a 44 años fueron los más afectados por la enfermedad de Tuberculosis Pulmonar, esto se debió a que en éste intervalo de edades mantuvo las mayores relaciones sociales (en el trabajo, amistades) por lo que estuvieron más expuestos a contraer la enfermedad, al igual que en los pacientes que trabajan de manera independiente (48.4\%) (13).

La interacción social con los miembros y organizaciones de la comunidad constituye un potencial fuente de apoyo para las personas. A partir de estas relaciones no sólo se pueden obtener importantes recursos, información y ayuda; sino también se deriva un sentimiento de pertenencia y de integración a una comunidad más amplia con importantes implicaciones para el bienestar individual y social. Paralas autoras los criterios que definen la integración social de una persona se encuentran, entre otros "estar en contacto regular con los vecinos", "tener amigos o conocidos en el vecindario" y la pertenencia a grupos sociales (14).

Las limitaciones laborales que genera la Tuberculosis Pulmonar se ve reflejada en varios casos, no es fácil tener la enfermedad y un trabajo a la vez, ello genera tensión en el paciente provocando cambios de humor; así mismo, algunos ponen de manifiesto que han dejado de trabajar o se han visto en la necesidad de escoger entre el trabajo y el tratamiento de la enfermedad durante el primer mes hasta que negativice (7).

Estudios realizados durante los últimos años han intentado medir el grado de soporte y/o apoyo a pacientes con enfermedades crónicas; sin embargo, este estudio pretende evaluar el impacto directo que tiene la familia y los amigos para que el paciente concluya satisfactoriamente con su tratamiento. El tratamiento, a diferencia de otras enfermedades crónicas, es de duración larga, con varios medicamentos cuyos efectos secundarios son visibles en la mayoría de los pacientes (13).

En la correlación de las variables del estudio, se evidencia que el soporte familiar y social se relacionan directamente con el cumplimiento del tratamiento de los pacientes con Tuberculosis Pulmonar; esto indica que a valores altos de soporte familiar y social corresponden valores altos de cumplimiento del tratamiento $(\mathrm{p}=0,002)$.

Se observa además que un $81.8 \%$ de los pacientes que recibieron un nivel alto de soporte familiar y social presentaron un alto nivel de cumplimiento del tratamiento; mientras que un $36,4 \%$ de los pacientes que tienen bajo nivel de soporte familiar y social presentaron bajo cumplimiento; se observa también que un $42,3 \%$ de los pacientes encuestados presentan un nivel de soporte familiar y social alto; mientras que un $21,2 \%$ presentan bajo nivel de soporte familiar y social.

Cuando el paciente percibe el apoyo, ayuda y preocupación de la familia, se sentirá más confiado y motivado a culminar con el tratamiento para poder recuperarse pronto y reinsertarse a su vida cotidiana,por otro lado, el apoyo social influye de manera directa en el paciente, ya que se sabe que la tuberculosis pulmonar daña su autoestima generando sentimientos de culpa, depresión, resentimiento y vergüenza, ya que son factores que van a influir negativamente en el adecuado cumplimiento del tratamiento $(7,10)$.

La Tuberculosis Pulmonar en la mayoría de los pacientes causa un "debilitamiento general", que se manifiesta con poca energía, agotamiento físico para el trabajo y estudio, así mismo, este debilitamiento se asocia a diferentes niveles de discapacidad por parte de los pacientes, con niveles variablesde perturbación de sus actividades productivas y sociales (7). Contar con soporte familiar o no va a tener algunas repercusiones importantes sobre la salud de las personas ya que ayudan a mantener la salud, amortiguan los efectos negativos que pueden producir ciertos acontecimientos de la vida. Por el contrario, la pérdida o ausencia de soporte en esencia por parte de la familia, asociada a diferentes fuentes de estrés potencian $\mathrm{y}$ crean un alto nivel de vulnerabilidad (15).

Según Weakland, las interacciones familiares son muy importantes tanto en el origen como en el curso y resultado de las enfermedades. Este autor señalaba que la familia poseía una influencia directa (pudiendo dificultar o facilitar funciones corporales) como indirecta (ayudando 
o interfiriendo en la aplicación de tratamientos médicos).

Por otro lado, Campbell y Treat, dicen que la familia puede ejercer un efecto sobre la salud de sus miembros a través de dos caminos: el psicofisiológico y el conductual.

Desde el punto de vista psicofisiológico, se hace referencia a los factores familiares relacionados con el estrés,si la persona cuenta con un buen funcionamiento de la familia, ésta se convierte en el principal centro de apoyo, en cambio si el funcionamiento de la familia es inadecuado, puede generar un estrés adicional.

Desde el punto de vista conductual, se hace referencia a las prácticas de salud que ejercen influencia sobre la persona. Así, algunos comportamientos relacionados con la salud pueden ser la dieta, el ejercicio, el descanso y sueño, por otro lado el uso de alcohol son, en parte, aprendidos y mantenidos dentro de la familia. Se ha destacado desde hace algunos años la importancia de la familia en el seguimiento de regímenes médicos, cambios de estilos de vida y participación en programas de rehabilitación (12).

El soporte social es fuente de amor, cuidado, afirmación y autoayuda en los pacientes con enfermedad crónica. Las redes sociales tienen un efecto directo sobre la salud mediante la interacción con los demás, proporcionando un sentido de identidad social, de pertenencia y participación, fomentando conductas saludables, incrementando la autoestima y favoreciendo el grado de adaptación a un estímulo estresante para obtener una repuesta positiva y satisfactoria al tratamiento de la enfermedad (15).

El soporte social actúa a manera de amortiguador entre los eventos estresantes de la vida y la enfermedad, hay evidencia entonces para sugerir que un cohesivo y suficiente soporte social podría favorecer el pronóstico de mejoría del paciente. El tener una red social tupida o un fuerte soporte social implica que el paciente tiene varios roles para fortalecerse a sí mismo y dar un sentido reforzante y positivo a su vida (17).

El paciente, lejos de ser un mundo autónomo y aislado, es también miembro de diversas redes sociales (el AA.HH, las juntas directivas, los vecinos, amigos, etc.) y forma parte de una cultura. Así mismo la unidad en la que, directa o indirectamente, repercuten las consecuencias de las crisis sociales, que la sociedad construye alrededor de enfermedades específicas (6).

Se puede deducir que un $59,6 \%$ de los pacientes encuestados si cumplen con el tratamiento; mientras que un $40,4 \%$ no presentan un cumplimiento adecuado.

Es muy común que un paciente afectado por la enfermedad considere que el tratamiento es muy difícil de mantener o continuar, expresando frecuentemente su deseo de abandonar el tratamiento debido a diversos factores, entre ellos: el exceso de tabletas que recibe cada día, los efectos adversos de los medicamentos, la pérdida de esperanza frente a la curación, los problemas económicos y/o familiares como consecuencia del tratamiento, motivos religiosos, etc., (7).

La Tuberculosis Pulmonar es curable, pero es necesario un diagnóstico temprano; pues es una enfermedad grave si no se cumple el tratamiento adecuado. En seguida, es indispensable no abandonar el tratamiento, al suspenderlo, la enfermedad se empeora rápidamente y causa que el bacilo se haga resistente a los medicamentos (13). El incumplimiento de las indicaciones médicas, no solo es grave porque hace ineficaz el tratamiento prescrito contra la Tuberculosis, sino que produce un aumento de la morbilidad y mortalidad; así como aumenta los costos de la asistencia sanitaria. Es así que genera grandes pérdidas en el aspecto personal, familiar y social (12).

Se entiende que el control de la tuberculosis, no solo depende de la prescripción de un tratamiento correcto de parte del personal sanitario sino también depende de las actitudes y voluntad propia del paciente de asumir las normas o consejos dados por el médico o personal sanitario, realizadas éstas tras una decisión completamente razonada (18). Éstas medidas que contribuyen al control de la enfermedad dependen de los hábitos aprendidos de cada paciente para fomentar su salud y prevenir el contagio de la enfermedad (7).

\section{REFERENCIAS BIBLIOGRÁFICAS}

1. Ahumada M, Escalante E, Santiago I. Estudio preliminar de las relaciones entre las estrategias de afrontamiento y el apoyo social con la adherencia al tratamiento de personas que viven con VIH/SIDA. Subjetividad y procesos cognitivos. 2011 (Citado el 04 de febrero del 2014); 15(1): 55-70. Disponible en: http://dspace.uces.edu.ar:8180/xmlui/ bitstream/handle/123456789/1046/Estudio_prel_Ahumada. pdf?sequence $=1$

2. Azzollini S, BailPupko V, Vida V. Diabetes: importancia de la familia y el trabajo en la adhesión al tratamiento/ Diabetes. Anu investig. 2011 (Citado el 10 de febrero del 2014);18: 323-330. Disponible en: http://www.scielo.org. ar/pdf/anuinv/v18/v18a35.pdf

3. Barra E. Apoyo social, estrés y salud. Psicología y Salud. 2004 (Citado el 04 de febrero de 2014); 14(2): 237-243. Disponible en: http://revistas.uv.mx/index.php/psicysalud/ 
article/view/848/1562

4. Bravo E. Actitud de los pacientes hacia su condición de multidrogorresistente en el Centro de Salud Fortaleza. Tesis Doctoral. Lima, Perú: Facultad de Medicina Humana, E.A.P. Enfermería, Universidad Nacional Mayor de San Marcos; 2008. (Citado el 06 de febrero del 2014). Disponible en: http://cybertesis.unmsm.edu.pe/bitstream/ cybertesis/538/1/bravo_ce.pdf

5. Cabrera L. Construyendo la agenda de tuberculosis de Lima Metropolitana. Lima: Consorcio Gráfico y Suministros; 2012. (Citado el 08 de febrero del 2014) Disponible en: http://www.tuberculosis.pe/sites/default/files/documentos/ agendatb_lima_metropolitana6.pdf

6. Contreras A. Factores que influyen en la adherencia a tratamientos farmacológicos y no farmacológicos en los pacientes inscritos en el Programa de Control de la Hipertensión Arterial de la Unidad Básica de Atención de COOMEVA. Salud Uni Norte. 2010 (Citado el 04 de febrero del 2014); 26(2): 201-211. Disponible en:http://www.scielo. org.co/pdf/sun/v26n2/v26n2a04.pdf

7. Fabelo J, Iglesias S, Bachir S, Ramírez A, Amador J. Percepción de apoyo social por pacientes con enfermedad alcohólica en fase de recuperación. Rev. Hosp. Psiquiátrico de la Habana.2012(Citado el 04 de febrero del 2014); 9(1). Disponible en: http://www.revistahph.sld.cu/rev12012/hph07112.html

8. Flores I. Adherencia al tratamiento en pacientes con factores de riesgo cardiovascular. Av enferm. 2009(Citado el 04 de febrero del 2014); 27(2): 25-32. Disponible en: http://www. enfermeria.unal.edu.co/revista/articulos/xxvii2 4.pdf

9. Heredia M, Puc M, Caamal A, Vargas A. Determinantes sociales relacionados con el tratamiento de tuberculosis. Revista Biomed. 2012 (Citado el 04 de febrero del 2014);23(3). Disponible en: http://www.medigraphic.com/ pdfs/revbio/bio-2012/bio123e.pdf

10. Martínez Y, Guzmán F, Flores J, Vázquez V. Factores familiares que favorecen el apego al tratamiento en casos de tuberculosis pulmonar. Aten Fam. 2014 (Citado el 04 de febrero del 2014); 21(2):47-49. Disponible en: http://www. medigraphic.com/pdfs/atefam/af-2014/af142e.pdf

11. Ministerio de Salud. Informe de la tuberculosis en el Perú. Lima: Ministerio de Salud; 2010 (Citado el 08 de febrero del 2014).Disponible en: http://www.minsa.gob.pe/portada/ Especiales/2011/respiravida/archivos/Ayuda_memoria Lanzamiento_TB.pdf

12. Organización de los Estados Americanos. Concepto de familia: la familia un sistema. Montevideo: Instituto interamericano del niño, la niña y adolescente; 2008. (Citado el 03 de febrero del 2014).Disponible en: http://www.iin.oea.org/Cursos_a_distancia/Lectura $\% 20$ 12_UT_1.PDF

13. Organización Mundial de la Salud. Tuberculosis. Ginebra: Organización Mundial de la Salud; 2014 (Citado el 10 de Enero del 2014). Disponible en: http://www.who.int/ mediacentre/factsheets/fs104/es/.

14. Organización Panamericana de la Salud. La tuberculosis en la región de américas. [En línea]. Washington:
Organización Mundial de la Salud; 2013 (Citado el 10 de Enero del 2014).Disponible en: http://www.paho. org/hq/index.php?option $=$ com_docman\&task $=$ doc view\&gid $=22954 \&$ Itemid

15. Jave O. La carga regional de tuberculosis. En: Jave $\mathrm{O} . \mathrm{Tb}$ en Perú: actualización breve. Lima: National Strategy of TB Peru; 2011(Citado el 02 de Enero del 2014). Disponible en: http://www.imtunmsm.epiredperu.net/ eventos/aniversario2011/cursotb/ponencias/01_Jave_TB Peru.pdf

16. Rufí G, García Rodríguez J, Solera J, Moreno S. Tuberculosis. Madrid: SEIMC;2009.(Citado el 05 de febrero del 2014). Disponible en: http://www.seimc.org/ contenidos/documentoscientificos/procedimientosclinicos/ seimc-procedimientoclinicoVII.pdf

17. Vega O, Gonzales D. Apoyo social: elemento clave en el afrontamiento de la enfermedad crónico. Enfermería Global. 2009 (Citado el 04 de febrero del 2014); 16: 1-11. Disponible en: http://revistas.um.es/eglobal/article/ viewFile/66351/63971?origin=publication_detail .

18. Vinaccia S, Quiceno J, Fernández H, et al. Calidad de vida, personalidad resistente y apoyo social percibido en pacientes con diagnóstico de cáncer pulmonar. Psicología y Salud. 2005 (Citado el 04 de febrero del 2014); 15(2): 207-

22. Disponible en: http://revistas.uv.mx/index.php/psicysalud/ article/view/805/1459

\section{Correspondencia:}

Sánchez Requin Ruth Liliana

Correo electrónico: Ruth.sanchez@upch.pe

Fecha de Recepción: 26 de enero de 2015. Fecha de aceptación: 25 de Junio de 2015. 